\title{
Virtual reality rehabilitation as a treatment approach for older women with mixed urinary incontinence: a feasibility study
}

\section{Authors}

Valérie Elliott,

Eling D. de Bruin,

Chantale Dumoulin

First published: 10 January 2014

\section{Funding Information}

Heinz Koelbl led the peer-review process as the Associate Editor responsible for the paper.

Conflict of interest: none.

\section{Abstract \\ Background}

Motivated patients are more likely to adhere to treatment resulting in better outcomes. Virtual reality rehabilitation (VRR) is a treatment approach that includes video gaming to enhance motivation and functional training.

Aims

The study objectives were (1) to evaluate the feasibility of using a combination of pelvic floor muscles (PFM) exercises and VRR (PFM/VRR) to treat mixed urinary incontinence (MUI) in older women, (2) to evaluate the effectiveness of the PFM/VRR program on MUI symptoms, quality of life (QoL), and (3) gather quantitative information regarding patient satisfaction with this new combined training program.

Methods

Women 65 years and older with at least 2 weekly episodes of MUI were recruited. Participants were evaluated two times before and one time after a 12-week PFM/VRR training program. Feasibility was defined as the participants' rate of participation in and completion of both the PFM/VRR training program and the home exercise. Effectiveness was evaluated through a bladder diary, pad test, symptom and QoL questionnaire, and participant's satisfaction through a questionnaire.

Results

Twenty-four women ( $70.5 \pm 3.6$ years) participated. The participants complied with the study demands in terms of attendance at the weekly treatment sessions (91\%), adherence to home exercise (92\%) and completion of the three evaluations (96\%). Post-intervention, the frequency and quantity of urine leakage decreased and patientreported symptoms and QoL improved significantly. Most participants were very satisfied with treatment (91\%). 


\section{Conclusion}

A combined PFM/VRR program is an acceptable, efficient, and satisfying functional treatment for older women with MUI and should be explore through further RCTs.

\section{INTRODUCTION}

Pelvic floor muscle (PFM) training has shown efficacy and is the recommended first-line treatment for patients with stress (SUI), urge (UUI), or mixed (MUI) urinary incontinence.[1] Both individualized PFM treatments and group-based exercise classes have been shown to be effective in treating these types of UI, even in older women.[2-4] However, despite the effectiveness of PFM training, the potential and sustained benefits are frequently hindered by poor patient adherence, both in the short- and long-term, and has been identified as an important predictor of overall PFM effectiveness. $[5,6]$

Adherence to a regular PFM exercise program is key to its initial effectiveness $[5,6]$ and poor adherence to a maintenance exercise program is responsible for a decline in effect.[5, 7, 8] However, despite the low level of difficulty, non-invasiveness, and efficacy of PFM training, high attrition rates, and low adherence are well documented in both clinical and research settings[9]; as a result, adherence has been identified as an influential short- and long-term predictor of PFM exercise effectiveness.[6, 10] Motivation is key to adherence as motivated patients are more likely to adhere to treatment.[11] Thus, one of the greatest challenges for PFM physiotherapists is identifying and incorporating techniques or approaches in their interventions that will promote patient adherence and, consequently, treatment effectiveness.

This study examined one such approach, virtual reality rehabilitation (VRR). VRR is a treatment approach that combines dynamic functional exercises (those that focus on lower body muscles and core stability to simulate common movements to prepare muscles for daily tasks) and video gaming in order to enhance motivation, thereby increasing participation, promoting adherence, and reducing dropout rates.[12] VRR employs computergenerated (virtual reality) environment using widely available game consoles (e.g., Nintendo Wii, Sony Playstation) to guide patients through clinician-prescribed interactive functional exercises in a fun and engaging manner.[13] Although many randomized control trials (RCTs) have demonstrated the effectiveness and motivational advantages of VRR-delivered therapies for various conditions and patient populations (e.g., those with stroke or balance problems),[14-16] no study has yet evaluated the effects of this novel treatment approach on urinary incontinence treatments and, more specifically, for older women with UI. Therefore, this feasibility study was necessary to determine if VRR (functional training) combined with PFM training (contractions) is a feasible treatment approach with older women with MUI; that is, would they participate in, comply to and complete the combine PFM/VRR program demands.

The objectives of this quasi-experimental pre-test, post-test pilot study were (1) to evaluate the feasibility of using a PFM/VRR program to treat MUI in older women, (2) to evaluate the effectiveness of the PFM/VRR program on $\mathrm{MUI}$ symptoms and quality of life (QoL), and (3) to gather quantitative information regarding patient satisfaction with the PFM/VRR training program. In the present study, feasibility is defined as the participants' rate of participation and completion for both the PFM/VRR training and the home exercise components. 


\section{MATERIALS AND METHODS}

Participants

Community-dwelling women were recruited through newspaper advertisements and promotional activities in seniors' organizations. Participants were included if they were female, aged 65 years and older, lived at home, were ambulatory (i.e., able to walk indoors/outdoors without any assisted devices), understood French or English (verbal and written instructions), had not changed their hormone prescription within the last 6 months, and suffer from MUI. MUI was defined by an affirmative answer to questions 2 and 3 of the short Urogenital Distress Inventory (UDI).[17] This validated indicator of UI has been previously used in non-experimental and randomized controlled trials. $[18,19]$ In addition, a history of urinary leakage secondary to urgency and physical exertion, coughing or sneezing, at least twice a week or more, within the last 3 months, was required for inclusion.

Participants were excluded if they had chronic constipation (per the Rome III criteria),[20] a pelvic organ prolapse of grade II or more according to the pelvic organ prolapse quantification system (POP-Q),[21] or perineal pain; were taking medication known to affect continence; or had mobility, medical or psychological problems that would interfere with the physical assessment or the PFM exercise program.

Participant eligibility using the above criteria was assessed by a questionnaire completed during a telephone interview with a research assistant.

The study received ethical approval from the Comité d'éthique de la recherche de l'Institut Universitaire de Gériatrie de Montréal and written consent was obtained from each participant prior to the first evaluation.

\section{Intervention Protocol}

Each woman participated in two pre-intervention evaluations (pre-1 \& pre-2) followed by a 12-week PFM/VRR training program and one post-intervention evaluation (post). The first two evaluations were conducted 2 weeks apart to ensure consistency in the incontinence measures prior to the intervention. They were identical; both included an interview using the same three questionnaires (UDI-6, the IIQ and the ICIQ-UI) and a modified 1-hr pad test.[22] However, demographic, medical and gynaecological history was only taken in the first evaluation (pre-1). All three evaluations were done by the same experienced physiotherapist (VF).

The 12-week intervention was delivered to groups of eight participants in a weekly 60-min exercise class under the supervision of another experienced physiotherapist (VE). Each session included a 10-min education period, a 30-min session of static PFM and deep abdominal (transverse abdominis [TrA]) training in different static positions as lying, sitting, four point kneeling and standing feet apart. The exercise class ended with a 20-min VRR training using a dance game, StepMania with dynamic PFM training. Participants were also given a 20-min static PFM home exercise program to do at home, 5 days per week. A weekly exercise diary was provided to each participant in which to record their home exercise participation. Details of the intervention protocol and progression are described in Appendix I.

The virtual reality component of the class session used a computer with a freeware dance game program, StepMania (www.stepmania.com), to create a virtual environment. Stimuli in the dance game were five instrumental songs paired with visual cues on how to dance each music track (i.e., arrows indicating step movements in four cardinal directions). A sequence of scrolling arrows that moved upwards on the screen cued 
each move (lower extremity movements); the participant had to make the indicated step on the dance mat when the relevant arrow reached the top of the screen. Different arrow sequences were generated for all five tracks. PFM contractions were represented by a red dot incorporated into the sequence of arrows as part of the combined functional PFM training exercise (see Fig. 1). In sum, the PFM strengthening component was provided through the addition of the PFM training component (i.e., PMF contractions) which preceded the VRR functional component (the step movements).

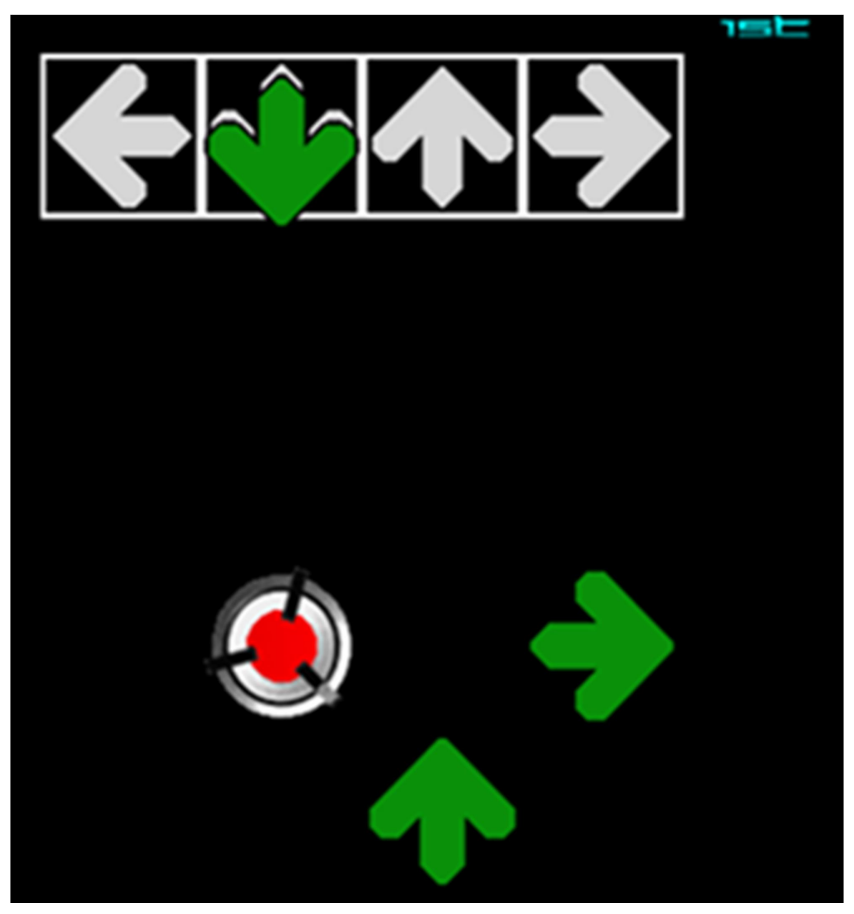

Figure 1. Example of the dance game series of arrows that included a PFM contraction represented by the red dot.

\section{Outcome Measurements}

Feasibility

Feasibility was defined as the participants' rate of participation and completion of both the PFM/VRR training and the home exercise programs. Class participation was calculated as the number of classes attended by each participant against the overall number of classes offered (a maximum of 12). Participants were required to complete at least 10 classes to be considered as having completed the intervention. The completion rate for the PFM/VRR training program was calculated as the proportion of participants who completed the final assessment. Participation in the home exercise program was calculated by summing up the adherence frequency for the home exercises over the 12-week period: participants were asked to record each time they completed the home exercises in their weekly exercise diary. Attrition was measured as the number of participants lost at follow-up.

Effectiveness of the intervention on MUI symptoms and QoL

The effectiveness of the PFM/VRR training program on MUI symptoms and on QoL was evaluated using measures of urinary leakage, frequency, and quantity; patient reported symptoms; and quality of life. 
To evaluate the frequency of UI leakage, the 72-hr (3-day) bladder diary was used. The diary included questions on the number of leakage episodes, provoking factors related to leakage, and the time and number of micturition, as well as the number of protections (sanitary pads) used during the 3-day period.

The usual 1-hr pad test[23] had to be adapted in order to include functional tests for older adults (see Appendix II). Thus to evaluate the quantity of urinary leakage, a modified 1-hr pad test was used with a standardized bladder volume, using a bladder scan; $300 \mathrm{ml}$ was the required minimum to proceed with the test. To accommodate for sweating and vaginal discharge, up to $2 \mathrm{~g}$ of weight gain in the pad test was considered an indication of continence.[22]

To measure patient-reported symptoms and QoL, the short form of the Urogenital Distress Inventory (UDI-6), the Incontinence Impact Questionnaire (IIQ), and the ICIQ-UI were used. All three assessed the presence of various urogenital symptoms and their effect on QoL.

The five outcome measures, the 72-hr bladder diary, the 1-hr pad test, the UDI-6, the IIQ and the ICIQ-UI, have been found to be valid and reliable measures of incontinence symptoms and UI-related QoL in women.[17, 22, 24-26]

\section{Participant satisfaction with treatment}

To evaluate participant's treatment satisfaction, a visual analogue scale (VAS) for satisfaction and a questionnaire were used at the end of the PFM/VRR intervention period. Participants' overall assessment of their satisfaction with the VRR component was recorded on a $10 \mathrm{~cm}$ VAS using the words "not satisfied" and "extremely satisfied."[27] Participants were also questioned regarding their satisfaction with the treatment received. Only two responses were possible: "satisfied," indicating that the participant did not want another treatment, and "dissatisfied," indicating that the participant wanted an alternative treatment to the one provided in the study.[3]

Perception of improvement

Participants' perception of improvement at the end of the intervention period was also measured using an Estimated Percent Improvement (EPI) question that rated the participant's improvement on a percentage scale.[28]

\section{Sample Size Justification and Statistical Analysis}

Based on previous work, a priori power calculation $(\alpha=0.05, \beta=0.90)$ was performed and determined that 20 participants were needed to detect a significant change in the pad test, mean UI episode/day for the 72-hr urinary diary and IIQ. The sample size was set at 24 , to allow for dropouts.

Analyses were performed with the SPSS software, version 19 (IBM SPSS Statistics, New York). The significance level was set at 0.05 for all tests. Descriptive analysis was used for class and home exercise participation, participant satisfaction with treatment outcomes, and perception of improvement. Data from the questionnaires and the 72-hr bladder diary were normally distributed; hence each of the outcome measures for the three evaluations (pre-1 \& -2 and post) were compared using repeated measures analysis of variance (ANOVAs). Data for the modified 1-hr pad test were not normally distributed, hence, were compared using the non-parametric 
repeated measures comparison (Friedman test). For effect size, we used $r$; suggested norms for interpreting $r$ are: $0.1=$ small, $0.3=$ moderate, and $0.5=$ large effect.[29]

\section{RESULTS}

\section{Participant Baseline Characteristics}

Twenty-four women participated in the study and had the following means and standard deviations (SD): age 70.5 (3.6) years, body mass index of $25.9(3.6) \mathrm{kg} / \mathrm{m} 2$, hysterectomy $0.7(0.5)$ and pregnancy 1.7 (1.4), including 1.3 (1.3) vaginal deliveries and $0.1(0.3)$ Caesarean sections.

Feasibility: Participation and Completion

The majority of participants complied with the study demands in terms of attendance at the weekly treatment sessions (91.0\%), adherence to the home exercise program (92.0\%) and completion of the three (pre-1, pre-2 and post) evaluations (96.0\%). The assessments and intervention were well tolerated, with an attrition rate of $4.0 \%$. Only one participant was unable to complete the physical part of the post-intervention evaluation due to an acute ankle injury not related to the intervention.

Effectiveness of Intervention on UI Severity and QoL

There was no significant difference between the pre-1 and pre-2 evaluations for the 72-hr urinary diary (mean voiding, leakage, and protections per day) nor in the UDI-6, IIQ, and ICIQ-UI scores, showing stability of UI and QoL measures in the absence of the intervention (Table I). Except for the mean protection measured in the 72-hr urinary diary, there was a significant improvement for all $\mathrm{UI}$ and QoL outcomes in the post evaluations as compared to both the pre- 1 and the pre- 2 evaluations (Table I).

Table I. UI PFM/VRR Responsiveness Outcomes Using Repeated-Measures ANOVA $(n=24)$

\begin{tabular}{|c|c|c|c|}
\hline Pre $-1^{1}$ mean \& SD & Pre $-2^{2}$ mean \& SD & Post $^{3}$ mean \& SD & Statistic test \& P-value \\
\hline \multirow[t]{4}{*}{$8.62(3.01)$} & $7.88(2.64)$ & $6.79(2.10)$ & $F(2.22) 19.39 P^{1} 1 / 40.000^{*}$ \\
\hline & & & $P^{1-2} \frac{1 / 4}{0.060}(95 \%$ IC $-0.023,1.496)$ \\
\hline & & & $P^{2-3} \frac{1 / 4}{40.008^{*}}(95 \%$ IC $0.258,1.937)$ \\
\hline & & & $P^{1-3} \frac{1}{4} 0.000^{*}(95 \%$ IC $-2.577,-1.090)$ \\
\hline \multirow[t]{4}{*}{$1.51(1.34)$} & $1.19(1.13)$ & $0.51(0.49)$ & $F(2,22) 8.93 P 1 / 40.001^{*}$ \\
\hline & & & $\mathrm{P}^{1-2} \frac{1 / 4}{4} 0.151(95 \%$ IC $-0.080,0.719)$ \\
\hline & & & $P^{2-3} 1 / 40.010^{*}(95 \%$ IC $0.143,1.218)$ \\
\hline & & & $P^{1-3} 1 / 40.001^{*}(95 \%$ IC $-1.597,-0.403)$ \\
\hline \multirow[t]{4}{*}{$0.90(0.81)$} & $0.77(0.90)$ & $0.47(0.88)$ & $F(2,22) 5.42 P^{1 / 4} 0.012^{x}$ \\
\hline & & & $P^{1-2} 1 / 40.525(95 \%$ IC $-0.11,0.36)$ \\
\hline & & & $P^{2-3} 1 / 40.125(95 \%$ IC $-0.06,0.67)$ \\
\hline & & & $P^{1-3} \frac{1}{4} 40.010^{*}(95 \%$ IC $-0.77,-0.09)$ \\
\hline \multirow[t]{4}{*}{$47.05(15.47)$} & $42.01(14.16)$ & $19.79(11.14)$ & $F(2,22) 30.53 P^{1 / 4} 0.000^{*}$ \\
\hline & & & $\mathrm{P}^{1-2} 1 / 40.448(95 \%$ IC $-3.68,13.75)$ \\
\hline & & & $\mathrm{P}^{2-3} \frac{1 / 4}{4} 0.000^{*}(95 \%$ IC $14.35,30.09)$ \\
\hline & & & $P^{1-3} 1 / 40.000^{*}(95 \%$ IC $-37.56,-16.96)$ \\
\hline \multirow[t]{4}{*}{$70.44(39.83)$} & $58.64(48.38)$ & $24.62(33.14)$ & $F(2,22) 10.41 P^{1 / 4} 0.001^{*}$ \\
\hline & & & $\mathrm{P}^{1-2} \frac{1 / 4}{0.205}(95 \%$ IC $-4.14,27.75)$ \\
\hline & & & $\mathrm{P}^{2-3} \frac{1}{4} 0.007^{*}(95 \%$ IC $8.40,59.63)$ \\
\hline & & & $P^{1-3} \frac{1}{4} 0.000^{*}(95 \%$ IC $-71.33,-20.32)$ \\
\hline \multirow[t]{4}{*}{$11.13(3.35)$} & $10.21(3.77)$ & $5.00(3.470)$ & $F(2,22) 24.51 P^{1 / 4} 0.000^{*}$ \\
\hline & & & $\mathrm{P}^{1-2} 1 / 40.871(95 \%$ IC $-1.27,3.10)$ \\
\hline & & & $P^{2-3} 1 / 40.000^{*}(95 \%$ IC $2.68,7.73)$ \\
\hline & & & $P^{1-3} \frac{1 / 4}{4} 0.000^{*}(95 \%$ IC $-8.38,-3.87)$ \\
\hline
\end{tabular}


Of the 24 participants, only 14 were able to reach a bladder volume of $300 \mathrm{ml}$ before starting the modified 1-hr pad test. However, secondary to an acute ankle injury, one of the 14 participants was unable to complete the physical part of the post-treatment evaluation. Applying a $\mathrm{P}=0.017$ Bonferroni adjustment, among those participants with a bladder volume $300 \mathrm{ml}$, the modified 1-hr pad test changed significantly between pre-1 and post $(n=13, T=0, P=0.008, r=-0.74)$ and pre-2 and post $(n=13, T=55, P=0.005, r=0.78)$, but not between pre-1 and pre- $2(n=13, T=-1, P=0.313, r=-0.28)$.

\section{Treatment Satisfaction}

According to the satisfaction VAS, the mean appreciation score of the VRR component in the training program was $9.8 / 10(0.5)$. The satisfaction question revealed that $91.0 \%$ of the participants were satisfied with the overall PFM/VRR training program and would not seek additional treatment.

\section{Perception of Improvement}

The mean percent improvement of the UI condition estimated by the participants was $72.3 \%$ (22.7\%). More than half of the group (54.0\%) reported $\geq 75.0 \%$ improvement; only one participant reported a low improvement of $15.0 \%$.

\section{DISCUSSION}

This feasibility study is the first to explore the use of VRR, which combines functional exercises and video gaming, as a complimentary component to PFM conventional rehabilitation. It demonstrated that women aged 65 and over, with MUI, are good candidates for a PFM/VRR training program and are capable of complying with study demands, as demonstrated by the high participation and low attrition rates.

In fact, all outcomes for responsiveness to the PFM/VRR training program showed a statistically significant improvement between the pre-2 evaluation, which took place just before the 12-week PFM/VRR training program, and the post-intervention evaluation (P-value range $[P=0.00-0.01])$. Further, the improvements in $\mathrm{UI}$ symptoms and in QoL are also supported by the absence of statistically significant changes in any of the responsiveness outcome measures ( $P$-value range $[P=0.06-0.87]$ ) between the first two evaluations, pre- 1 and pre-2.

When evaluating the validity of a study it is important to consider both the clinical and statistical significance of the findings.[30] Studies that claim clinical relevance may lack sufficient statistical significance to make meaningful statements or, conversely, may lack practicality despite showing a statistically significant difference in treatment options. Researchers and clinicians should not focus on small P-values alone to decide whether a treatment is clinically useful; it is necessary to also consider the magnitude(s) of treatment differences and the power of the study.[30] Encouraging in this context is the observation that the majority of the pre-post intervention comparisons showed high magnitude(s) for the observed differences. The "minimum clinically important difference" (MCID) was reached for each of the responsiveness outcomes between the pre-2 and post evaluations. The MCID for the mean UI episodes, using the $72 \mathrm{hr}$ bladder diary, is a $50.0 \%$ reduction[31]; the study showed a $58.0 \%$ reduction. Similarly, the MCID for the UDI-6 score is a 10-point reduction[32]; this study demonstrated a 22-point decrease. The MCID for the IIQ score is a decrease of 10-25 points[33]; the study showed a 34-point decrease. Additionally, the study found a five-point reduction in the ICIQ-UI score; the MCID is only a two-point reduction.[34] Finally, the MCID for the pad test is a 50.0\% reduction in urinary leakages[35]; 
the present study showed an $85.0 \%$ reduction. Therefore, the PFM/VRR training program was effective in reducing UI symptoms and enhancing QoL to a clinically significant degree.

Four studies, one cohort and three RCTs on PFM training groups, used PFM exercises classes to treat older women with $\mathrm{UI}$, hence, can be compared to the present study.[2-4]

Interestingly, the mean attendance rate to the weekly treatment sessions for the present study (91.0\%) was higher than that obtained by the Dumoulin cohort study (82.2\%) as well as that of Kim and collaborators (2007) in their RCT (82.4\%). Further, the completion rate of the present study $(96.0 \%)$ was also higher than those obtained by the Dumoulin study (80.8\%) and the RCTs conducted by Kim (2007), Pereira (2011), and Sherburn (2011; 88.0-95.0\%). While these differences may, in part, be attributable to the VRR component, they could also be linked to differentials in age, UI type and treatment delivery with the present study. At 65 and older, the participants in the present study were the same age as in the Sherburn study, but slightly younger than those in Kim's (70+) and slightly older than those in Dumoulin's (60+) and Pereira's (60+). While this study focused exclusively on MUI, Dumoulin's study focused on both MUI and SUI, and the remaining studies-Sherburn, Pereira, and Kim-focused exclusively on SUI. Finally, the frequency of treatment, hence an increased participation burden, was also higher in Kim's study (twice a week) compared to this study (once per week). As suggested by the comparative results, the VRR program may have enhanced treatment adherence and completion rates; however, slight variations between this study's and the four studies' protocols render a degree of uncertainty, which needs to be explored through further randomized control trials (RCTs).

In addition to the above, variations in incontinence outcomes were also noted, but, again, these could also be attributable to variations in the data collection methods. Following treatment, Perreira's RCT (2011), which used the 1-hr pad test,[23] recorded an average reduction of $1.42 \mathrm{~g}(\mathrm{n}=17)$ compared to $31.13 \mathrm{~g}$ for the present study $(n=13)$. However, while both used a 1-hr pad test, the present study adapted Pereira's pad test to include functional fitness tests (the results of which have been presented in a scientific abstract[36]); specifically, the walking component at the beginning of the test was divided into standing functional fitness tests and the stair climbing component was replaced by more walking and an additional minute of light running.

As in this study, Sherburn's RCT (2011) also used the ICIQ-UI to measure the presence of various urogenital symptoms and their effects on QoL. Sherburn noted a mean post-treatment improvement of 4.5 points compared to 5.21 points for the present study. The intervention protocols for both studies included education periods and strengthening exercises for pelvic floor and abdominal muscles in different positions, but only this study used VRR. Both Dumoulin's and this study included the IIQ and a 72-hr bladder diary. The mean reduction (\%) in the number of daily UI episodes post-treatment was $48.0 \%$ in Dumoulin's study compared to $58.0 \%$ in the present study. For the IIQ, the mean improvement post-treatment was 16.83 points for Dumoulin's study, compared to 34.02 points for the present study. Although, not as dynamic as the VRR component, Dumoulin's intervention also included trunk and leg strengthening sessions.

Thus, it is possible that the VRR component, specifically the expert level which required participants to contract their PFMs while doing a dance move, helped participants in the present study to develop their ability to contract their PFMs during physical activities, which could, in part, explain the difference in pad testing (with Perreira's) and the ensuing functional improvements reflected in both the ICIQ-UI (Serburn) and IIQ (Dumoulin) scores. However, it is impossible to reach a definitive conclusion without further RCTs. 
Noteworthy and further supporting the need for more research on VRR, the $91 \%$ satisfaction rate among participants in the present study was somewhat higher than that obtained in the group and individual treatments in Pereira's RCT, 86\%. Further, the VRR component of the training program was highly appreciated by all study participants as demonstrated by a mean satisfaction VAS scores of 9.8 over 10 . The addition of the VRR component offers a potential explanation for the increased satisfaction rating, which may have also facilitated treatment adherence but, again, requires further testing as a hypothesis.

Even more interestingly, the mean estimated percent of UI-condition improvement in the present study was $72.33 \%$, which was higher than the actual mean reduction in incontinence episodes, 58\%, documented in the 72 hr urinary diary. In other words, participants' subjective rating for percent improvement was higher than the actual improvement. Participants' estimated percentage of improvement seems to not only reflect actual improvements in the frequency of urinary leakages, as reported in their diaries, but may also reflect an increased confidence in their ability to self-manage their UI. Thus, the estimated percent of improvement, as reported by participants, suggests that beyond the reduction or elimination of urinary leakages, there may be other factors equally important to UI management, such as confidence or the ability to deal with everyday situations that could cause urinary leakage, which the VRR component may have helped to address.

According to the results of this feasibility study, the advantages of integrating VRR to a conventional PFM training are:[1] it seems to increase short-term adherence and participation by introducing a game-like aspect to the training program and[2] introducing PFM contractions into the virtual reality dance game appears to help participants to develop their ability to contract PFMs during movement. This latter has useful applications to everyday situations, such as retaining urine while walking to the toilet. Another potential advantage of the combined PFM/VRR program, which has not been discussed in this manuscript, is its potential beneficial effect in terms of improved executive functions, which is interesting as recent evidence suggests that older women with MUI demonstrate a decline in executive-function.[37] Furthermore, it could also be beneficial in improving dualtask gait in older women with MUI. The effects of the PMF/VRR program on executive functions and dual-task gait in older women with $\mathrm{MUI}$ are described in detail by Fraser et al. in The effects of combined pelvic floor muscle training and virtual-reality dance rehabilitation on dual-task gait and cognition in women with mixedurinary incontinence, submitted recently to the Arhcives of physical therapy.

Limitations of the Study and Further Research

As this was a feasibility study, the sample size was limited and methodology did not include a control group; hence, the clinical applications of this study are limited. However, the positive results found in this study, in terms of feasibility, effectiveness, and participant's appreciation of the combined intervention, support the need for an RCT to compare this novel PFM/RRV training program to the absence of treatment and, eventually, standard conventional PFM training programs for the treatment of $\mathrm{UI}$ in elderly women. Based on the results of this study, minor modification should be made in the study outcomes of further RCTs. The quantity of urinary leakage should be measured by a 24-hr pad test in order to eliminate the lengthy and difficult process of bladder filling engendered by the 1-hr test. As we have hypothesized that the PFM/RRV training program could have impacted confidence with its functional component, self-efficacy outcome measures such as the Brooms questionnaire should be included in future studies.[38] The treatment protocol should remain the same as it was appreciated by the participants. Future RCTs should also include long-term follow-up to evaluate whether the treatment effects of PFM/VRR training programs can be sustained over time, in particularly as adherence is not only a problem during the intervention phase but also post-intervention. Finally, the data for this feasibility 
study should be used to calculate the sample size of the future RCT comparing conventional PFM trainings with and without VRR.

\section{CONCLUSION}

A combined PFM/VRR is an acceptable functional training approach for older women with MUI, one in which they are capable of complying with treatment program demands. Not only is it effective in reducing UI symptoms and in enhancing QoL but has a high level of patient satisfaction. This new approach, combining dynamic functional exercises and video gaming, could potentially increase participant adherence to UI treatment programs; however, this remains to be tested.

\section{ACKNOWLEDGMENT}

The authors wish to thank Vanessa Faro-Dussault, project assessor as well as the participants for their commitment in this study. 


\section{Appendix I: Intervention Protocol}

TABLE AI. Topics Discussed During the Education Period of the Weekly Class

Week 1

Week 2

Week 3

Week 4

Anatomy of the PFM

Pathophysiology of UI

Risks factors of U

Constipation

prevention Definition of $\mathrm{U}$

Evacuation technique

Week 5

Week 6

Week 7

Week 8

Foods that irritate the bladder

Hydration

Advice to suppress urges

Bladder retraining program

Week 9

Week 10

Week 11

Week 12 
PFM and deep

abdominal (TrA)

exercises

component

\begin{tabular}{|c|c|c|c|c|}
\hline $\begin{array}{l}\text { PFM } \\
\text { maximal } \\
\text { contraction }\end{array}$ & $\begin{array}{l}3 \text { sets of } 8 \\
\quad \text { contractions } \times 6\end{array}$ & $\begin{array}{l}3 \text { sets of } 10 \\
\quad \text { contractions } \times 8\end{array}$ & $\begin{array}{l}3 \text { sets of } 12 \text { contractions } \\
\times 10 \text { sec }\end{array}$ & $\begin{array}{l}\text { Standing, sitting, } \\
\text { and supine }\end{array}$ \\
\hline The knack & $\begin{array}{l}3 \text { sets of } 3 \text { cycles of one } \\
\text { PFM contraction, cough } \\
\text { and PFM relaxation }\end{array}$ & $\begin{array}{l}3 \text { sets of } 3 \text { cycles of one } \\
\text { PFM contraction, cough, } \\
\text { and PFM relaxation }\end{array}$ & $\begin{array}{l}3 \text { sets of } 3 \text { cycles } \\
\text { of one PFM contraction, } \\
\text { cough and PFM } \\
\text { relaxation }\end{array}$ & Standing \\
\hline The podium & $\begin{array}{l}3 \text { podiums; } 6 \mathrm{sec} \\
\text { per level }\end{array}$ & 3 podiums; $8 \mathrm{sec}$ per level & $\begin{array}{l}3 \text { podiums; } \\
10 \text { sec per level }\end{array}$ & Sitting \\
\hline TrA contraction & $\begin{array}{l}\text { Supine knee flexed, } \\
1 \text { set of } 10 \text { TrA } \\
\text { contractions } \times 5 \mathrm{sec} \\
\text { combined with } \\
\text { alternating hip } \\
\text { extension }\end{array}$ & $\begin{array}{l}\text { Supine knee flexed, } \\
1 \text { set of } 10 \mathrm{TrA} \\
\text { contractions } \times 5 \mathrm{sec} \\
\text { combined with a sit-up }\end{array}$ & $\begin{array}{l}\text { Supine knee flexed, } \\
1 \text { set of } 10 \mathrm{TrA} \\
\text { contractions } \times 5 \mathrm{sec} \\
\text { combined } \\
\text { with alternating } \\
\text { diagonal sit-up }\end{array}$ & Supine knee flexed \\
\hline $\begin{array}{l}\text { TrA contraction } \\
\text { against gravity }\end{array}$ & $\begin{array}{l}\text { Four point kneeling, } \\
1 \text { set of } 10 \mathrm{TrA} \\
\text { contractions } \times 5 \mathrm{sec}\end{array}$ & $\begin{array}{l}\text { Four point kneeling, } \\
1 \text { set of } 10 \mathrm{TrA} \\
\text { contractions } \times 5 \mathrm{sec} \\
\text { combined with alternating } \\
\text { arms elevation }\end{array}$ & $\begin{array}{l}\text { Four point kneeling, } \\
1 \text { set of } 10 \mathrm{TrA} \\
\text { contractions } \times 5 \mathrm{sec} \\
\text { combined with } \\
\text { alternating } \\
\text { legs elevation }\end{array}$ & Four point kneeling \\
\hline VRR component & $\begin{array}{l}\text { Beginner level: } 4 \text { songs } \\
\text { One foot at a time }\end{array}$ & $\begin{array}{l}\text { Intermediate level } 4 \\
\text { songs The right and left } \\
\text { foot are simultaneously } \\
\text { not doing the same } \\
\text { dance step }\end{array}$ & $\begin{array}{l}\text { Expert level: } 4 \text { songs Idem } \\
\text { intermediatepred dot } \\
\text { representing a } \\
\text { PFM contraction }\end{array}$ & Standing dancing game \\
\hline
\end{tabular}

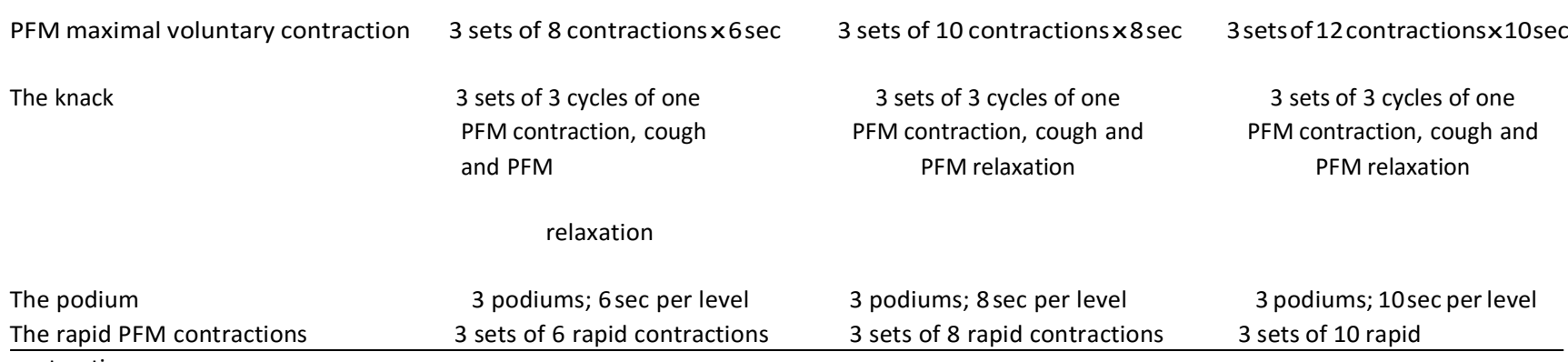




\section{Appendix II: The Modified $1 \mathrm{hr}$ Pad Test}

- Women had to drink $500 \mathrm{ml}$ of water $1 \mathrm{hr}$ before the evaluation.

- At the beginning of the evaluation, bladder quantity was measured by ultrasound (Bladder scan 3000, Diagnostic Ultrasound) and a minimum of $300 \mathrm{ml}$ was necessary to proceed with the modified 1-hr pad test.

The modified $1 \mathrm{hr}$ pad test sequence was as followed:

- three timed up and go tests,

- two 2-min walk tests,

- three repetitions of the unipodal stance test on the dominant leg,

- 10 repetitions of sit to stand from a toilet,

- picking up five objects from the ground,

- 1 min of hand washing,

- two sets of $1 \mathrm{~min}$ of light running,

- $30 \mathrm{~min}$ of walking with and without a cognitive task.

\section{REFERENCES}

1. Dumoulin C, Hay-Smith J. Pelvic floor muscle training versus no treatment, or inactive control treatments, for urinary incontinence in women. Cochrane Database Syst Rev 2010; CD005654.

2. Kim H, Suzuki T, Yoshida Y, et al. Effectiveness of multidimensional exercises for the treatment of stress urinary incontinence in elderly community-dwelling Japanese women: A randomized, controlled, crossover trial. J Am Geriatr Soc. [Randomized Controlled Trial Research Support, Non-U. S. Gov't] 2007; 55:1932-9.

3. Pereira VS, Correia GN, Driusso P. Individual and group pelvic floor muscle training versus no treatment in female stress urinary incontinence: A randomized controlled pilot study. Eur J Obstet Gynecol Reprod Biol $2011 ; 159: 465-71$.

4. Sherburn M, Bird M, Carey M, et al. Incontinence improves in older women after intensive pelvic floor muscle training: An assessor-blinded randomized controlled trial. Neurourol Urodyn 2011; 31:20968.

5. Chen HY, Chang WC, Lin WC, et al. Efficacy of pelvic floor rehabilitation for treatment of genuine stress incontinence. J Formos Med Assoc 1999; 98:271-6.

6. Kim H, Yoshida H, Suzuki T. The effects of multidimensional exercise on functional decline, urinary incontinence, and fear of falling in community-dwelling elderly women with multiple symptoms of geriatric syndrome: A randomized controlled and 6-month follow-up trial. Arch Gerontol Geriatr 2011; 52:99-105.

7. Alewijnse D, Mesters I, Metsemakers J., et al. Predictors of intention to adhere to physiotherapy among women with urinary incontinence. Health Educ Res 2001; 16:173-86.

8. Lagro-Janssen T, van Weel C. Long-term effect of treatment of female incontinence in general practice. $\mathrm{Br} \mathrm{J}$ Gen Pract 1998; 48:1735-8.

9. Kincade JE. A pilot study to determine reasons for patient withdrawal from a pelvic muscle rehabilitation program for urinary incontience. J Appl Gerontol 1999; 18:379-96.

10. Chen SY, Tzeng YL. Path analysis for adherence to pelvic floor muscle exercise among women with urinary incontinence. J Nurs Res 2009; 17:83-92. 
11. Sarma S, Hawthorne G, Thakkar K, et al. The development of an incontinence treatment motivation questionnaire for patients undergoing pelvic floor physiotherapy in the treatment of stress incontinence. Int Urogynecol J Pelvic Floor Dysfunct 2009; 20:1085-93.

12. De Bruin ED, Schoene D, Pichierri G, et al. Use of virtual reality technique for the training of motor control in the elderly. Some theoretical considerations. Z Gerontol Geriatr 2010; 43:229-34.

13. Van Schaik $P$, Blake J, Pernet $F$, et al. Virtual augmented exercise gaming for older adults. Cyberpsychol Behav 2008; 11:103-6.

14. Grealy MA, Johnson DA, Rushton SK. Improving cognitive function after brain injury: The use of exercise and virtual reality. Arch Phys Med Rehabil 1999; 80:661-7.

15. Yavuzer G, Senel A, Atay MB, et al. "Playstation eyetoy games" improve upper extremity-related motor functioning in subacute stroke: A randomized controlled clinical trial. Eur J Phys Rehabil Med 2008; 44:237-44.

16. Saposnik G, Teasell R, Mamdani M, et al. Effectiveness of virtual reality using Wii gaming technology in stroke rehabilitation: A pilot randomized clinical trial and proof of principle. Stroke 2010; 41:1477-84.

17. Shumaker SA, Wyman JF, Uebersax JS, et al. Health-related quality of life measures for women with urinary incontinence: The Incontinence Impact Questionnaire and the Urogenital Distress Inventory. Continence Program in Women (CPW) Research Group. Qual Life Res 1994; 3:291-306.

18. Fantl JA, Wyman JF, Anderson RL, et al. Postmenopausal urinary incontinence: Comparison between nonestrogen-supplemented and estrogen-supplemented women. Obstet Gynecol 1988; 71:823-8.

19. Burgio KL, Goode PS, Locher JL, et al. Behavioral training with, without biofeedback in the treatment of urge incontinence in older women: A randomized controlled trial. JAMA 2002; 288:2293-9.

20. Longstreth GF, Thompson WG, Chey WD, et al. Functional bowel disorders. Gastroenterology 2006; 130:1480-91.

21. Haylen BT, de Ridder D, Freeman RM, et al. An International Urogynecological Association (IUGA)/International Continence Society (ICS) joint report on the terminology for female pelvic floor dysfunction. Neurourol Urodyn 2010; 29:4-20.

22. Jorgensen L, Lose G, Andersen JT. One-hour pad-weighing test for objective assessment of female urinary incontinence. Obstet Gynecol 1987; 69:39-42.

23. Abrams P, Blaivas JG, Stanton SL, et al. The standardisation of terminology of lower urinary tract function. The International Continence Society Committee on Standardisation of Terminology. Scand J Urol Nephrol Suppl 1988; 114:5-19.

24. Wyman JF, Fantl JA, McClish DK, et al. Comparative efficacy of behavioral interventions in the management of female urinary incontinence. Continence Program for Women Research Group. Am J Obstet Gynecol 1998; 179:999-1007.

25. Avery K, Donovan J, Peters TJ, et al. ICIQ: A brief and robust measure for evaluating the symptoms and impact of urinary incontinence. Neurourol Urodyn 2004; 23:322-30.

26. Uebersax J, Wyman J, Shumaker S, et al. Short forms to assess life quality and symptom distress for urinary incontinence in women: The Incontinence Impact Questionnaire and the Urogenital Distress Inventory. Continence Program for Women Research Group. Neurourol Urodyn 1995; 14:131-9. 
27. Stach-Lempinen EKP, Laippala R, Metsänoja B. Visual analogue scale, urinary incontinence severity score and $15 \mathrm{D}-$ Psychometric testing of three different health-related quality-of-life instruments for urinary incontinent women. Scand J Urol Nephrol 2001; 35:476-83.

28. Burgio KL, Goode PS, Richter HE, et al. Global ratings of patient satisfaction and perceptions of improvement with treatment for urinary incontinence: Validation of three global patient ratings. Neurourol Urodyn 2006; 25:411-7.

29. Cohen J. Statistical power analysis for the behavioural sciences. 2nd edition. Lawrence Erlbaum Associate.

30. Bhardwaj SS, Camacho F, Derrow A, et al. Statistical significance and clinical relevance: The importance of power in clinical trials in dermatology. Arch Dermatol 2004; 140:1520-3.

31. Yalcin I, Peng G, Viktrup L, et al. Reductions in stress urinary incontinence episodes: What is clinically important for women? Neurourol Urodyn 2010; 29:344-7.

32. Lin SN, Klapper AS, Wong P, et al. Quality of life after treatment with midurethral sling and concomitant prolapse repair in patients with mixed versus stress urinary incontinence. Neurourol Urodyn 2011; 30:150711.

33. Dyer KY, Xu Y, Brubaker L, et al. Minimum important difference for validated instruments in women with urge incontinence. Neurourol Urodyn 2011; 30:1319-24.

34. Sherburn M, Bo K, Galea M. Evaluation of outcome measures for stress urinary incontinence in older women. Neurourol Urodyn 2009; 28:715-6.

35. US DoHaHS, Administration FaD, Health CfDaR. Guidance for industry and FDA Staff-clinical investigations of devices indicated for the treatment of urinary incontinence. 2011 [updated 05/16/2011]; Available from: http://www.fda.gouv/medicaldevices/deviceregulationandguidancedocuments/ucm070852.htm\#ft_18

36. Elliott V, Fraser $\mathrm{S}$, Chaumillon J, et al. The effect of virtual reality rehabilitation on the gait parameters of older women with mixed urinary incontinence: A feasibility study. Neurourol Urodyn 2012; 31:883.

37. Lussier M, Renaud M, Chiva-Razavi S, et al. Are stress and mixed urinary incontinence associated with impaired executive control in community-dwelling older women? J Clin Exp Neuropsychol 2013; 35:445-54.

38. Broome BA. The impact of urinary incontinence on self-efficacy and quality of life. Health Qual Life Outcomes 2003; 1:35.

This is the accepted version of the following article Elliott V, de Bruin E, Dumoulin C. (2015) Virtual reality rehabilitation as a treatment approach for older women with mixed urinary incontinence: a feasibility study. Neurourol Urodyn. 2015 Mar; 34(3). 236-243., which has been published in final form at http://onlinelibrary.wiley.com/doi/10.1002/nau.22553/epdf 